\section{The podocyte adhesome}

Loss of podocyte adhesion is a major contributing factor to the progression of glomerular disease; however, comprehensive understanding of the role of focal adhesions (FAs) in podocyte biology is lacking. New research has identified the FERM-domain protein, EPB41L5, as an important podocyte FA protein with roles in actomyosin contractility, FA maturation, cell spreading, migration and extracellular matrix (ECM) sensing. "Identification of key components of the native podocyte adhesome and understanding the role of specific regulators of podocyte adhesion opens new avenues to understand and potentially target glomerular diseases," says Tobias Huber.

To identify key components of the podocyte adhesome, Schell, Rogg, Huber and colleagues isolated podocyte cell populations from murine glomeruli and used quantitative mass spectrometry to identify nearly 3,500 podocyte proteins. A series of filtering steps to enrich for FA proteins led to the identification of EPB41L5. "To understand the podocytespecific role of EPB41L5 we combined conditional knockout alleles in mice, super resolution microscopy, genome editing and in vitro focal adhesion proteomics," says Huber.

Podocyte-specific deletion of Epb4115 led to podocyte detachment, proteinuria and glomerulosclerosis in mice, together with altered localization and decreased expression of key podocyte proteins, suggesting a role in podocyte maturation. To further assess the role of EPB41L5, the researchers generated EPB41L5-deficient immortalized human podocyte cell lines, which showed defective migration and adhesion when plated on an ECM substrate. They also observed pseudopods, characterized by an unorganized actin cytoskeleton, in spreading EPB41L5-knockout podocytes, suggesting impaired actomyosin function likely caused by dysregulation of the GTPases, RhoA and Rac1. Finally, the researchers identified a role for EPB41L5 in ECM sensing and signalling to modulate the FA composition, highlighting a key role for EPB41L5 in maintaining the integrity of the glomerular filtration barrier. Susan J. Allison

Identification
of key
components
of the native
podocyte
adhesome ...
opens new
avenues to
understand
and potentially
target
glomerular
diseases
\footnotetext{
EPB41L5 regulates actomyosin contractility and focal adhesion formation to maintain the kidney filtration barrier. Proc. Natl Acad. Sci. USA http://dx.doi.org/10.1073/
}

ORIGINAL ARTICLE Schell, C. et al. The FERM protein pnas.1617004114 (2017) 